\title{
Gestão de RH e processos de recrutamento \& seleção e integração nas cooperativas de crédito através da metodologia DISC durante pandemia Coronavírus
}

\section{HR management and recruitment \& selection and integration processes in credit cooperatives through DISC methodology during Coronavirus pandemic}

DOI: $10.46814 / \operatorname{lajdv2n3-001}$

Recebimento dos originais: 10/03/2020

Aceitação para publicação: 10/03/2020

\section{Jaciara Xavier Pereira Ribeiro}

Doutoranda em Ciências da Educação pela Instituição Brasil de Educação e Pesquisa - IBEP, em parceria com a Universidade Nacional de La Plata e Universidade Del Sol

Instituição: Instituição Brasil de Educação e Pesquisa - IBEP, em parceria com a Universidade Nacional de La Plata e Universidade Del Sol - UNADES/IBEP/BRASIL

Endereço: UNADES - 14 De Mayo 462, Asunción, Paraguai/ IBEP - sede no Calçadão Arthur Bernardes, $n^{\circ}$ 152, Sala 06, Bairro Centro, Viçosa - MG - CEP 36.570- 061

E-mail: jaciaraxp@yahoo.com.br

\section{RESUMO}

A Gestão de Recursos Humanos e dos Processos de Recrutamento \& Seleção e integração das Pessoas nas Cooperativas de Crédito SICOOB, através da metodologia DISC durante pandemias, a exemplo do coronavirus, também conhecido como Covid-19, sendo um campo de estudo muito importante pois está relacionado ao fator humano, muito importante para promover o sucesso da cooperativa de crédito. Sendo assim, para a definição do problema de pesquisa levou em consideração a expectativa de investigar a importância do Gestor em se capacitar para a realização de uma Gestão eficiente e eficaz do Setor de Recursos Humanos e dos Processos de Recrutamento \& Seleção e integração das Pessoas nas Cooperativas de Crédito SICOOB, com a utilização da metodologia DISC para promover o triunfo das instituições financeiras cooperativas de crédito. Tendo como objetivo geral Apresentar conceitos importantes na área de Gestão de Pessoas e Processos de Recrutamento \& Seleção e Integração das Pessoas nas Instituições financeiras Cooperativistas, tendo como objetivos específicos: Apresentar Metodologia DISC aplicada na Gestão de Pessoas, nos Processos de Recrutamento \& Seleção e Integração e Gestão de Crise econômica em Decorrência da Pandemia; Apresentar conceitos sobre a Cooperativismo; Demonstrar o perfil de colaboradores de uma cooperativa pesquisada. A cooperativa apresenta em seu quadro maioria do sexo feminino, com alto grau de escolaridade e motivação pelo trabalho. Os objetivos foram alcançados, respondendo à questão problema. Como limitação do artigo, a metodologia utilizada para pesquisa foi feita apenas revisão documental e bibliográfica, neste sentido, sugere-se a realização de novas pesquisas, desta vez de forma quantitativa ou qualitativa em outras instituições financeiras e organizações financeiras

Palavras-chave: Gestão, Cooperativismo, DISC, Recrutamento, Seleção.

\begin{abstract}
The Management of Human Resources and Recruitment \& Selection and Integration of People in Credit Cooperatives SICOOB, through the DISC methodology during pandemics, like the coronavirus, also known as Covid-19, being a very important field of study because it is related to the human factor, very important to promote the success of the credit union. Therefore, in order to define the research problem, it
\end{abstract}


took into account the expectation of investigating the importance of the Manager in training for the achievement of an efficient and effective Management of the Human Resources Sector and of the Recruitment \& Selection and Integration processes of People in Credit Unions SICOOB, using the DISC methodology to promote the triumph of credit cooperative financial institutions. With the general objective to present important concepts in the area of People Management and Recruitment \& Selection and Integration of People in Cooperative Financial Institutions, having as specific objectives: To present DISC Methodology applied in People Management, in the Recruitment \& Selection and Integration Processes and Management of Economic Crisis Due to Pandemic; Present concepts about Cooperativism; Demonstrate the profile of employees of a researched cooperative. The cooperative is mostly female, with a high level of education and motivation for work. The objectives were achieved, answering the problem question. As a limitation of the article, the methodology used for research was made only documentary and bibliographic review, in this sense, it is suggested to carry out new research, this time in a quantitative or qualitative way in other financial institutions and financial organizations

Keywords: Management, Cooperativism, DISC, Recruitment, Selection.

\section{INTRODUÇÃO}

A Gestão de Recursos Humanos e dos Processos de Recrutamento \& Seleção e integração das Pessoas nas Cooperativas de Crédito SICOOB, através da metodologia DISC durante pandemias, a exemplo do coronavirus, também conhecido como Covid-19, sendo um campo de estudo muito importante pois está relacionado ao fator humano, muito importante para promover o sucesso da cooperativa de crédito.

Importante que gestores com formação em Bacharel em Administração ou Bacharel em Ciências Contábeis, busquem formação em especializações no curso de Pós-Graduação em Psicologia Organizacional e Psicopedagogia Clinica e Institucional, para promover nos níveis estratégico, tático e operacional das organizações cooperativistas, conhecimento e preparo para lidar com as pessoas internas envolvidas com as cooperativas e com as pessoas externas que desejam ingressar no quadro de funcionários, através dos processos de recrutamento e seleção e na integração deste novo(a) colaborador(a) dentro das cooperativas. Mapear o Perfil Comportamental dos colaboradores é uma forma de enaltecer o trabalho, ajudando cada pessoa a encontrar seu lugar de maneira feliz e confortável. Isso é promover o desenvolvimento humano.

A Metodologia MODELO DISC - iniciais de dominância, influência, estabilidade e conformidade - Moderno sistema de identificação de Perfil comportamental, através de testes como Profiler e DISC. Sendo assim, para a definição do problema de pesquisa levou-se em consideração a expectativa de investigar a importância do Gestor em se capacitar para a realização de uma Gestão eficiente e eficaz do Setor de Recursos Humanos e dos Processos de Recrutamento \& Seleção e integração das Pessoas nas Cooperativas de Crédito SICOOB, com a utilização da metodologia DISC para promover o triunfo das instituições financeiras cooperativas de crédito. 
O problema é expresso, então, pela questão: qual a importância da gestão eficiente e eficaz dos Departamento de Recursos e Humanos e processos de Recrutamento \& Seleção e Integração das Pessoas as Cooperativas de Crédito? Tendo como objetivo geral: Apresentar conceitos importantes na área de Gestão de Pessoas e Processos de Recrutamento \& Seleção e Integração das Pessoas nas Instituições financeiras Cooperativistas, tendo como objetivos específicos: Apresentar Metodologia DISC aplicada na Gestão de Pessoas, nos Processos de Recrutamento \& Seleção e Integração e Gestão de Crise econômica em Decorrência da Pandemia; Apresentar conceitos sobre a Cooperativismo; Demonstrar o perfil de colaboradores de uma cooperativa pesquisada;

Vergara (2005, p.32) aborda que o autor de um estudo o justifica ao atribuir-lhe contribuições de ordem prática ou ao estado da arte na área. Neste sentido o presente trabalho poderá trazer como contribuição prática, o conhecimento sobre a A Gestão do Setor de Recursos Humanos e dos Processos de Recrutamento, Seleção e integração das Pessoas nas Cooperativas de Crédito SICOOB.

A metodologia utilizada quanto aos meios de investigação, para a realização deste estudo, tornou-se necessário o desenvolvimento de uma pesquisa documental e de caráter bibliográfica, conceituando termos relacionados aos conceitos gerais sobre os temas tratados.

Este artigo está estruturado em capítulos, incluindo a introdução, na qual se apresenta o problema de pesquisa, os objetivos, a justificativa, metodologia e a estrutura do artigo. O segundo capítulo é desenvolvimento, no qual são apresentadas as referências teóricas. No terceiro formulam-se as conclusões, acrescidas das limitações e sugestões para novos estudos, e por fim são apresentadas as referências bibliográficas.

\section{DESENVOLVIMENTO}

A tarefa dos profissionais da gestão de pessoas é de suma importância para a organização e para todos os colaboradores que atuam na mesma, pois, segundo Knapik (2012).

\footnotetext{
A área ou departamento de gestão de pessoas é o conjunto de políticas e subsistemas que se preocupa com o gerenciamento estratégico das pessoas e leva à eficácia dos colaboradores em busca dos objetivos pessoais e empresariais (KNAPIK, 2012, p.53).
}

O contexto de gestão de pessoas envolve dois polos distintos: pessoas e organizações. As pessoas passam grande parte de seu tempo nas organizações, dependem destas, não apenas para ganharem o salário que garanta sua subsistência, mas também sucesso e realização pessoal. Como é uma via de mão dupla, a organização depende dessas pessoas para funcionarem e, consequentemente, atingirem seus objetivos e alcançarem sucesso. Percebe-se, assim, uma relação simbiótica entre as organizações e as pessoas (CHIAVENATO, 2010). 
Os objetivos da área da gestão de pessoas são proporcionar aos colaboradores motivação e engajamento; desenvolver programas de treinamento e desenvolvimento profissional; assegurar à empresa uma vantagem competitiva; manter a qualidade de vida dos trabalhadores; gerenciar mudanças; assegurar uma política ética e de abertura; e, desenvolver lideranças (KNAPIK, 2010).

A gestão de pessoas é indispensável não apenas ao sucesso, mas ao funcionamento de uma organização e é uma área que vem sofrendo uma série de transformações com o passar dos anos. Segundo Chiavenato (2010, p.6), "falar de gestão de pessoas é falar de gente, de mentalidade, de inteligência, de vitalidade, ação e pró-ação". A gestão de pessoas sinaliza claramente como o fator humano continua importante e indispensável às organizações, mesmo na era da informação, marcada pelo surgimento de novas tecnologias que, a princípio, parecem valorizar a máquina em detrimento do humano.

\footnotetext{
Gestão de Pessoas é o conjunto integrado de atividades de especialistas e de gestores - como agregar, aplicar, recompensar, desenvolver, manter e monitorar pessoas - no sentido de proporcionar competências e competitividade à organização. Gestão de Pessoas é a área que constrói talentos por meio de um conjunto integrado de processos e cuida do capital humano das organizações; o elemento fundamental do seu capital intelectual é a base do seu sucesso (CHIAVENATO, 2010, p.9).
}

Ao invés de investir diretamente em produtos e serviços, as grandes organizações têm voltado seus investimentos para as pessoas - profissionais que entendem de produtos e serviços e, por isso, mostram-se capazes de criá-los, desenvolvê-los, produzi-los e ainda melhorá-los. "Neste mesmo caminho, ao invés de investirem nos clientes há um movimento de investir nas pessoas que os atendem, para que essas saibam satisfazê-los e encantá-los” (CHIAVENATO, 2010, p.4).

\subsection{METODOLOGIA DISC APLICADA NA GESTÃO DE PESSOAS}

DISC é uma sigla para quatro perfis de comportamento: dominância, influência, estabilidade e conformidade. Segundo Solides (2020), na década de 1920, William Marston, PhD em Psicologia pela Universidade de Harvard, elaborou a teoria DISC para explicar as reações emocionais dos seres humanos. Em seu livro "As emoções das pessoas normais", ele descreve os quatro tipos comportamentais principais, os quais originaram a expressão DISC:

- Dominance (dominância): remete ao controle, poder e assertividade.

- Influence (influência): relacionada à comunicação e às relações sociais.

- Steadiness (estabilidade): diz respeito à paciência e persistência

- Conscientiousness (cautela): relativa à organização e à estrutura.

Para Solides (2020), uma das adequações, por exemplo, é em relação à nomenclatura dos perfis, já que a mera tradução não passa a ideia clara do que é principal em cada um dos quatro. Assim, com a 
evolução da metodologia, trabalha-se com as seguintes nomenclaturas, respectivamente correspondentes aos perfis DISC: Executor; Comunicador; Planejador; Analista. Todas as pessoas possuem características dos quatro perfis comportamentais. Geralmente, uma pessoa possui um ou dois perfis cujas tendências de comportamento aparecem com mais frequência sendo perfis predominantes. O conhecimento dos quatro perfis comportamentais relacionados à Metodologia DISC nos dá insumos valiosos para a gestão de pessoas. O teste DISC retorna estilo de liderança, competências, áreas de talento no qual o trabalho ganha uma excelente ferramenta de análise preditiva e de desenvolvimento humano.

\subsubsection{Metodologia DISC nos Processos de Recrutamento \& Seleção e Integração}

Provavelmente, o uso mais comum da Metodologia DISC está no processo de recrutamento e seleção, já que muitas pessoas buscam por uma ferramenta para mapear o perfil de candidatos que se inscrevem em processos seletivos pois a assertividade da metodologia para prever padrões de comportamento é altíssima (SOLIDES, 2020).

Segundo Solides (2020), em um processo seletivo, o mapeamento comportamental também é um aliado no que diz respeito ao tempo. Se você aplica o teste em todos os inscritos para uma vaga, já consegue iniciar o seu processo com uma triagem comportamental. Basta desenhar a engenharia do cargo antes do processo e acabou a loteria de usar uma hora do seu dia para entrevistar alguém que passou apenas da triagem de currículos. Sabemos muito bem que essa pessoa pode ser muito diferente do perfil desejado e o encontro acaba sendo tempo perdido. Como o teste DISC pode ser respondido online e em casa, além do sistema guardar os dados do cargo e fazer a triagem, você não tem nenhum trabalho adicional, mesmo recrutando um número bem maior de candidatos.

Para Solides (2020), a metodologia DISC potencializa também a qualidade do processo seletivo. Ao sabermos as tendências comportamentais de alguém, conseguimos fazer uma entrevista mais personalizada e direcionada para os pontos críticos, por exemplo. Isso significa que você pode conhecer mais a fundo o candidato e que aumentam suas chances de acertar nessa vaga. E não podemos nos esquecer das seleções internas. A metodologia DISC também embasa decisões de mudança de cargo dentro de uma mesma empresa. Ou seja, ela traz assertividade para que a pessoa certa esteja no lugar certo. E isso significa mais produtividade e retenção de talentos. O conhecimento sobre os quatro perfis DISC permite que uma simples capacitação possa se tornar um treinamento comportamental.

A partir do momento em que entendemos como cada perfil aprende e o que potencializa o aprendizado, garantimos que o conteúdo seja passado e absorvido. Analisar o perfil da turma e personalizar sua atuação para evitar olhares desatentos e tempo perdido. A Metodologia DISC tem como base a valorização das características principais de cada perfil. Se o seu cargo te exige algo completamente diferente do que é fácil e natural para você, talvez essa seja a função errada. O 
conhecimento sobre perfis traz essa clareza de característica e nos permite entender em quais pontos de desenvolvimento vale a pena investir energia e quais exigências não fazem sentido para cada tipo (SOLIDES, 2020).

Segundo Solides (2020), é claro que falar que alguém tem um perfil dominante não significa colocar a pessoa em uma caixinha e dizer que ela jamais mudará. Por isso a evolução da metodologia DISC foi importante, já que agregou, entre outras teorias, o conhecimento sobre competências. A mesma lógica se aplica a qualquer competência e a qualquer outro perfil. Em resumo, a Metodologia DISC nos permite traçar caminhos inteligentes de desenvolvimento de pessoas. Além disso, algumas ferramentas trazem métricas que possibilitam uma verificação mais objetiva do processo de desenvolvimento, o que é uma grande aposta nos chamados Plano de Desenvolvimento Individual ou PDI's.

Quando pensamos em desenvolver líderes, o mesmo raciocínio do Plano de Desenvolvimento Individual se aplica. Considerando que o teste DISC conta com dados sobre estilo de liderança, esse tema pode ser diretamente acompanhado no desenvolvimento, inclusive (SOLIDES, 2020).

Porém, há uma vantagem extra: a Metodologia DISC é acessível aos líderes. Eles conseguem, com o suporte dos profissionais de gestão de pessoas, entender e gerir a sua equipe, de forma que se tornam cada vez mais emancipados nesse sentido. O relatório sobre cada colaborador traz informações que pessoas de todas as áreas conseguem compreender e a Gestão Comportamental é um caminho objetivo que guia as lideranças para de fato exercerem o papel de gestores de pessoas. E sabemos que essa autonomia das lideranças faz parte do caminho para uma atuação mais estratégica dos RH's (SOLIDES, 2020).

Segundo Solides (2020), ao termos conhecimento sobre os perfis, aprendemos também qual é a melhor maneira de dar feedbacks para cada um, tanto os positivos quanto os negativos. Os comunicadores, por exemplo, precisam de feedbacks constantes, mesmo se for sobre um ponto de atenção, já que podem, em meio ao seu otimismo, se esquecer dos aspectos negativos levantados. Os analistas, por outro lado, exigem muito de si mesmos, de forma que uma vez dado um feedback negativo, eles sempre se lembrarão. O cuidado deve ser justamente para não insistir nesses pontos. Similarmente, existem diversas outras dicas para que cada perfil realmente absorva o que será dito e se sinta confortável nesse processo. Isso vale para os feedbacks da avaliação de desempenho, para conflitos pontuais e até para o reconhecimento dos colaboradores. Saber valorizar alguém da maneira que é interessante para ela, e não para você, também é gerir pessoas de forma inteligente.

People Analytics é uma das maiores tendências atuais em RH, e significa simplesmente fazer análises com os dados sobre pessoas para embasar decisões e coletar indicadores. Aliado à Metodologia DISC é possível, por exemplo, mapear diversos padrões importantes para a gestão de pessoas. As aplicações da Metodologia DISC no uso dos perfis comportamentais na gestão do desempenho e 
performance, no clima organizacional, nos momentos de mudança organizacional, na resolução de conflitos, nas ações de endomarketing...Basicamente, em qualquer cenário de gestão de pessoas (SOLIDES, 2020).

Para Solides (2020) a partir do momento em que a Gestão Comportamental, por meio da Metodologia DISC, está intrínseca à sua gestão de pessoas, todos os processos de RH se otimizam. Com isso, as demais áreas da empresa sentem o impacto positivo e, certamente, a organização passa a ter um diferencial competitivo, já que ganha em produtividade, assertividade e satisfação dos colaboradores.

\title{
2.1.2 Gestão de Crise econômica em Decorrência da Pandemia Coronavírus e Metodologia DISC
}

Para Solides (2020), diante de um cenário de crise econômica em decorrência da pandemia do novo Coronavírus, preservar a atividade empresarial, tanto quanto possível, deve ser o foco principal de um gestor. Nesse momento, conciliar a gestão de crise e o Profiler Sólides é uma maneira eficiente de manter a estabilidade dos seus negócios. Sendo assim, os colaboradores são os responsáveis pelo reposicionamento e retomada da empresa. Logo, utilizar o Profiler, um teste de perfil comportamental, baseado na Metodologia DISC, que mapeia as principais tendências de comportamento das pessoas.

\begin{abstract}
O Profiler auxiliará, tanto o gestor como o setor de RH, na hora de entender como cada um desses perfis tendem a se comportar em situações de crise — o que é fundamental para elaborar ações e estratégias eficientes para manter produtividade e engajamento da equipe como um todo. O perfil comunicador tende a ser mais vaidoso, gostando de receber feedbacks positivos e reconhecimento pelo seu trabalho, o isolamento social que o momento demanda, pode ocasionar uma queda em sua energia, impactando na produtividade, pois pessoas desse grupo necessitam da coletividade, além de terem dificuldade de se organizar e priorizar tarefas. Já os planejadores, que também têm perfil relacional e gostam de coletividade, tendem a se sentirem com a energia mais baixa por causa do isolamento social. Isso porque, em home office, colaboradores desse grupo, quando se veem sozinhos, podem achar que não estão contribuindo de forma efetiva para a equipe. Os analistas são adeptos do ditado: "É melhor prevenir do que remediar". Por isso, o maior impacto sofrido por esse grupo em uma crise está na imprevisibilidade. Por serem pessoas que gostam de ter um controle do desenvolvimento da história, conhecendo os próximos passos, a falta dessa previsibilidade assusta. Os executores, por serem multitarefas e intuitivos, precisam se manter ativos, em uma rotina não muito engessada. Em contrapartida, as soluções propostas por eles podem não ter uma visão ampla e sistêmica, sendo imediatista (SOLIDES, 2020).
\end{abstract}

Para Solides (2020, no relatório do Profiler, existem algumas comparações entre os 4 perfis comportamentais e alguns animais específicos. Conseguir conciliar a gestão de crise e o Profiler ajuda na decisão sobre demissões e remanejamentos. Dentre essas medidas estão: quais profissionais serão desligados, que entrarão de férias e quantos deles precisam continuar na equipe para manter o funcionamento do negócio. O Profiler é uma ferramenta essencial na gestão de crise da Cooperativa. Além dessas informações, a análise de perfil comportamental proporciona outros índices, gráficos e relatórios completos. Tantos dados relevantes ajudarão na condução do seu negócio em qualquer situação que esteja vivendo. 


\subsection{COOPERATIVISMO}

O cooperativismo é tido como um dos principais instrumentos para a promoção de desenvolvimento econômico e social, pois gera e distribui renda e promove o capital social nas comunidades que o praticam (BIALOSKORSKI NETO, 2002, p.77:97).

O cooperativismo está embasado em sete princípios, quais sejam: adesão voluntária e livre, gestão democrática pelos membros, participação econômica dos membros, autonomia e independência, educação, formação e informação, intercooperação, interesse pela comunidade. (STEFANO; ZAMPIER; GRZESZCZESZYN, 2006). Estes princípios veem se modificando ao longo dos tempos. A Organização das Cooperativas Brasileiras (OCB) classifica as cooperativas do Brasil em treze ramos distintos: agropecuário, consumo, crédito, educacional, especial, habitacional, infraestrutura, mineral, produção, saúde, trabalho, transporte e turismo e lazer (OCEMG, 2011, p.8:9). Com destaque ao ramo crédito que segundo Pinheiro (2008, p.7), as cooperativas de crédito são instituições financeiras constituídas sob a forma de sociedade cooperativa, tendo por objetivo a prestação de serviços financeiros aos associados.

As cooperativas de crédito vêm crescendo muito no sistema financeiro e tem buscado no mercado, os melhores profissionais, encontrados através de realização de processos de recrutamento e seleção e integração do novo profissional na Instituição, que presta os mesmos serviços financeiros que os bancos, tendo em seus princípios as principais diferenças entre as partes. "o capital humano enquanto elemento detentor da aprendizagem organizacional passa a ser o diferencial, e com ele a necessidade cada vez maior das organizações de aprender e de desenvolver novos conhecimentos (FLEURY \& OLIVEIRA JUNIOR, 2002 apud NASCIMENTO, 2008)".

\subsubsection{Perfil dos Colaboradores da Cooperativa de Crédito Pesquisada}

A Seguir será apresentado o perfil dos colaboradores da Cooperativa de Crédito de Livre Admissão dos Vales do Piranga e Matipó Ltda - Sicoob União dos Vales, baseada na pesquisa documental desenvolvimento de minha Tese de Doutorado, no qual apresentou em março de 2020, um quadro de colaboradores de $\mathbf{2 1 8}$ funcionários, sendo $\mathbf{7 0 , 6 \%}$ do sexo feminino e $\mathbf{2 9 , 4 \%}$ do sexo masculino, no qual 17,9\% apresentam idade entre 18 a 24 anos, 56,9\% entre 25 a 34 anos; $\mathbf{2 2 , 5 \%}$ entre 35 e 44 anos; 2,7\% entre 45 e 59 anos. Quanto a Cor/Etnia segundo Classificação do IBGE, 52,8\% são de cor branca, 38,1\% são pardos, $6 \%$ são negros, $\mathbf{2 , 8 \%}$ amarelo, $\mathbf{0 , 3 \%}$ não declarado. Quanto ao estado civil, $\mathbf{5 3 , 2 \%}$ são casados, $\mathbf{3 8 , 5 \%}$ são solteiros, 4,6\% União estável, 3,7\% divorciados. 61,5\% não tem filhos; 3,2\% apresentam alguma deficiência. Em relação a nível de escolaridade dos colaboradores, observou-se que $\mathbf{0 , 5 \%}$ mestrado e/ou doutorado, $\mathbf{4 2 , 2 \%}$ possuem pós-graduação completa, $\mathbf{6 , 4 \%}$ com pós-graduação em andamento, 30,3\% possuem superior completo, 16,5\% superior incompleto, $\mathbf{4 , 1 \%}$ médio completo. Em relação a ANBIMA, 41,7\% possuem certificação. Tais resultados comprovam o alto 
nível em relação a formação acadêmica, sendo um dos motivos de destaque da Cooperativa que ocupa as melhores colocações no Ranking Financeiro do Sicoob Central Crediminas no Estado de Minas Gerais/BRASIL.

A Cooperativa pesquisada denominada Sicoob União dos Vales, possui uma Política de Recrutamento e Seleção aprovada pelo Conselho de Administração, sendo critério obrigatório a participação e aprovação, para a contração, sendo que $\mathbf{4 5 \%}$ do colaboradores contratados, declaram ter tido acesso pela primeira vez aos conceitos cooperativistas através de processo de recrutamento e seleção, provando que o evento pode também ser considerado, não somente uma ação para recrutar e selecionar, mas também, como um eficiente Programa de Educação Cooperativistas (PEC), pois divulga a todos os participantes a Doutrina e princípios Cooperativistas, entre outros conceitos e informações importantes relacionadas ao Cooperativismo, no qual o participante selecionado para contratação já se integra na organização dotado dos conceitos específicos da Instituição e os candidatos não selecionados, apesar de não obter a vaga de imediato, ficando no banco de reservas da Instituição, leva consigo conhecimento sobre o cooperativismo, fortalecendo a ação adotada pela cooperativa para difusão do Cooperativismo. Em relação aos Inventários metodologia DISC, $\mathbf{7 9 , 8 \%}$ dos funcionários, concordam com o resultado de seu perfil comportamental apresentado através dos relatórios do Profiler e feedback realizada pelo analista certificado na Instituição, sendo que $89 \%$ encontram-se motivados profissionalmente dentro da Instituição.

\section{CONCLUSÃO}

Para a realização deste artigo foi necessária uma revisão documental e bibliográfica sobre o tema abordado. Sendo assim, para a definição do problema de pesquisa levou-se em consideração a expectativa de investigar a importância da gestão eficiente e eficaz dos Departamento de Recursos e Humanos e processos de Recrutamento \& Seleção e Integração das Pessoas as Cooperativas de Crédito. O objetivo geral foi atingido sendo apresentado conceitos importantes na área de Gestão de Pessoas e Processos de Recrutamento \& Seleção e Integração das Pessoas nas Instituições financeiras Cooperativistas, sendo também atingidos os objetivos específicos no qual foi apresentado a Metodologia DISC aplicada na Gestão de Pessoas, nos Processos de Recrutamento \& Seleção e Integração e na Gestão de Crise econômica em decorrência da Pandemia sendo também apresentado os conceitos sobre a Cooperativismo com a demonstração do perfil dos colaboradores da cooperativa pesquisada.

Nota-se pelos resultados apresentados que a Cooperativa possui um alto nível de qualificação profissional, no qual 95,9\% dos funcionários concluíram curso superior ou está em andamento. Apenas 4,1\% apresentam ensino médio, sendo que 41,7\% possuem certificação ANBIMA. O SICOOB União dos Vales, está entre as maiores cooperativas financeiras, ocupando excelente colocação no ranking 
financeiro, tendo como entre muitas justificativas pelo Sucesso a qualificação de seus profissionais e níveis acadêmicos de seus gestores e colaboradores. A participação e aprovação de processos de Recrutamento \& Seleção são obrigatórias para a contração, sendo que $45 \%$ dos colaboradores contratados, declaram ter tido acesso pela primeira vez aos conceitos cooperativistas através de processo de recrutamento e seleção, provando que o evento pode também ser considerado, não somente uma ação para recrutar e selecionar, mas também, como um eficiente Programa de Educação Cooperativistas (PEC). 79,8\% dos funcionários, concordam com o resultado de seu perfil comportamental e 89\% encontram-se motivados profissionalmente dentro da Instituição. A metodologia utilizada quanto aos meios de investigação, para a realização deste estudo foi a pesquisa documental e de caráter bibliográfica, no qual foi apresentado os conceitos propostos. Diante dos resultados apontados e por tratar-se de um estudo constituído de apenas pesquisa bibliográfica e documental, pôde-se perceber que o artigo traz como limitação, a impossibilidade do levantamento da totalidade das pesquisas realizadas com relação ao tema tratado. Neste sentido, sugere-se a realização de novas pesquisas, desta vez de forma quantitativa ou qualitativa, por meio de questionários ou entrevista, com vistas a ampliar as análises dos resultados desta pesquisa em outras Instituições financeiras e organizações empresariais.

\section{REFERÊNCIAS}

BIALOSKORSKI NETO, Sigismundo. Gestão agroindustrial. GEPAI: Grupo de Estudos e Pesquisas Agroindustriais. São Paulo: Atlas, 1997.

CHIAVENATO, Idalberto. Gestão de pessoas: o novo papel dos recursos humanos nas organizações. Rio de Janeiro: Elsevier, 2010.

KNAPIK, Janete. Gestão de pessoas e talentos. Curitiba: Ibpex, 2012.

NASCIMENTO, Cláudia Terra do. O Psicopedagogo e a Aprendizagem Organizacional: A Importância da Gestão do conhecimento na Administração de Recursos Humanos. São Paulo, 2008. Disponível em Acesso em: 13 jan. 2010.

ORGANIZAÇÃO E SINDICATO DAS COOPERATIVAS DE MINAS GERAIS. Informações econômicas e sociais do cooperativismo mineiro. Belo Horizonte: Gerência Técnica do Sistema Ocemg/Sescoop-MG. 2011.

PINHEIRO, Marcos Antônio Henriques. Cooperativas de crédito: história da evolução normativa no Brasil.6. ed. Brasília: Banco Central do Brasil, 2008.

SOLIDES. Etologia e o profiler. Disponível em: https://blog.solides.com.br/etologia-e-o-profiler/ Acesso em 22 de abril de 2020. 
SOLIDES. Gestão de Crise e profiler em: https://blog.solides.com.br/gestao-de-crise-e-profiler/Acesso em 22 de abril de 2020.

SOLIDES. Metodologia-Disc. Disponível em: https://blog.solides.com.br/metodologia-disc/. Acesso em 22 de abril de 2020.

STEFANO, S.R. ZAMPIER, M. A.; GRZESZCZESZYN, G. Cooperativas: características, gestão e relevância sócio- econômica para o Brasil. In: SEMINÁRIOS EM ADMINISTRAÇÃO - SEMEAD, 9, 2006, São Paulo. Anais... (São Paulo: [s.n.], 2006, 16p.

VERGARA, Sylvia Constant. Projeto e relatórios de pesquisa em administração. São Paulo: Atlas, 2005. 